\title{
The Blackbody Radiation Spectrum Follows from Zero-Point Radiation and the Structure of Relativistic Spacetime in Classical Physics
}

\author{
Timothy H. Boyer \\ Department of Physics, City College of the City \\ University of New York, New York, New York 10031
}

\begin{abstract}
The analysis of this article is entirely within classical physics. Any attempt to describe nature within classical physics requires the presence of Lorentz-invariant classical electromagnetic zero-point radiation so as to account for the Casimir forces between parallel conducting plates at low temperatures. Furthermore, conformal symmetry carries solutions of Maxwell's equations into solutions. In an inertial frame, conformal symmetry leaves zero-point radiation invariant and does not connect it to non-zero-temperature; time-dilating conformal transformations carry the Lorentz-invariant zero-point radiation spectrum into zero-point radiation and carry the thermal radiation spectrum at non-zero temperature into thermal radiation at a different non-zerotemperature. However, in a non-inertial frame, a time-dilating conformal transformation carries classical zero-point radiation into thermal radiation at a finite non-zero-temperature. By taking the no-acceleration limit, one can obtain the Planck radiation spectrum for blackbody radiation in an inertial frame from the thermal radiation spectrum in an accelerating frame. Here this connection between zero-point radiation and thermal radiation is illustrated for a scalar radiation field in a Rindler frame undergoing relativistic uniform proper acceleration through flat spacetime in two spacetime dimensions. The analysis indicates that the Planck radiation spectrum for thermal radiation follows from zero-point radiation and the structure of relativistic spacetime in classical physics.
\end{abstract}




\section{INTRODUCTION}

Although the universal spectrum of blackbody radiation has historically been connected with statistical mechanics and quantum theory, the present article makes the connection with zero-point radiation and the structure of relativistic spacetime. It is suggested that classical thermal radiation in a general static coordinate system is the one-parameter stationary spectrum of isotropic random classical radiation obtained from the scale-invariant spectrum of zero-point radiation by the time-dilating conformal transformation which preserves the wave equation and carries radiation normal modes into normal modes. [1] [2] Although it is suggested that this definition of thermal radiation can be extended to a general spacetime, it is illustrated here only for a scalar radiation field in flat spacetime in two spacetime dimensions. It is pointed out that an inertial frame is such a specialized coordinate frame that a time-dilating conformal transformation is identical with a space-dilating conformal transformation, and that zero-point radiation in an inertial frame remains invariant under all conformal transformations and so does not allow us to obtain the blackbody spectrum at non-zero temperature from the Lorentz-invariant zero-point radiation spectrum. However, the thermal radiation spectrum in an inertial frame can be obtained by going to a relativistic accelerating frame (a Rindler frame), applying a time-dilating conformal transformation to zero-point radiation in that coordinate frame, and then carrying the spectrum back to an inertial frame by taking the no-acceleration limit. Work related to the present article has been published in the Physical Review D[1] and in the American Journal of Physics[2]; however, in these articles, the connection with the conformal group is absent.

The work described here is completely at variance with the suggestions of the physicists in the early 20th century that classical physics leads inevitably to the Rayleigh-Jeans spectrum as the spectrum of blackbody radiation. [3] The physicists of that period were aware neither of classical zero-point radiation nor of the importance of relativity and so used nonrelativistic statistical mechanics or scattering of radiation by nonrelativistic nonlinear systems when determining the spectrum of thermal equilibrium. In the present article, we are replacing these nonrelativistic calculations by symmetry considerations in relativistic spacetime. In line with the suggestions here regarding the importance of relativistic physics for radiation equilibrium, it has been shown that the zero-point radiation spectrum is invariant under scattering by the relativistic scattering system of a relativistic charged particle in a Coulomb 
potential.[4] This result is again in complete contrast with the scattering results involving nonrelativistic nonlinear mechanical systems. [5]

\section{PRELIMINARIES}

\section{A. Conformal Symmetry and Fundamental Constants}

Electromagnetic theory is invariant not only under Lorentz transformations but also under the still larger group of conformal transformations. [6] The conformal transformations in an inertial frame include both the scale-changing dilatations (which will be used in the present article), as well as the special conformal transformations which correspond to local changes of the spacetime scale.[7] Sometimes the dilatations of the conformal group have

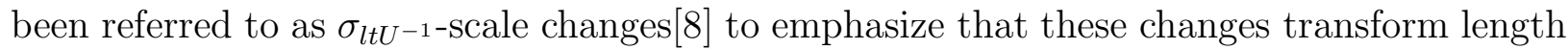
and time with the same multiplicative constant while transforming the energy with the inverse of the multiplicative constant. Thus in an inertial frame, if under a dilatation the length $l$ is mapped to $\bar{l}=\sigma l$ where $\sigma$ is a positive real number, then the time is mapped as $t \rightarrow \bar{t}=\sigma t$, and the energy $U$ mapped as $U \rightarrow \bar{U}=U / \sigma$. Thus the dilatations of the conformal group preserve the values of the fundamental constant $c$ (the speed of light in vacuum, involving length divided by time), the constant $a_{S} / k_{B}^{4}$ (Stefan's constant of blackbody radiation divided by Boltzmann's constant to the fourth power, involving the inverse third power of energy times length), and the constant $e$ (the charge of the electron, involving the square-root of energy times length).

\section{B. Metrics}

In this article, we will discuss not electromagnetic radiation but rather the analogue system of relativistic scalar radiation in two spacetime dimensions in flat spacetime. In an inertial frame, the coordinates $x^{0}=c t, x^{1}=x$ can be chosen as geodesic coordinates and the spacetime metric $d s^{2}=g_{\mu \nu} d x^{\mu} d x^{\nu}$ takes the Minkowski form

$$
d s^{2}=d x^{0} d x^{0}-d x^{1} d x^{1}=c^{2} d t^{2}-d x^{2}
$$

Thus in an inertial frame, the time coordinate $t$ is connected to the interval length by the universal constant $c$. 
A Rindler coordinate frame [9] in flat spacetime involves time-constant proper acceleration at each point of the static coordinate frame. We can pass from an inertial frame to a Rindler frame by relating the time and space coordinates $c t, x$ in an inertial frame to $\eta, \xi$ in the Rindler frame as

$$
\begin{aligned}
& c t=x^{0}=\xi \sinh \eta \\
& x=x^{1}=\xi \cosh \eta
\end{aligned}
$$

The metric is then given by

$$
d s^{2}=c^{2} d t^{2}-d x^{2}=\xi^{2} d \eta^{2}-d \xi^{2}
$$

In this metric involving $\eta$ and $\xi$, the constant $c$ no longer appears explicitly and the time parameter $\eta$ does not carry any units.

\section{Scalar Radiation Field}

In an inertial frame, the Lagrangian for a massless relativistic real scalar field $\phi$ is given by

$$
\mathcal{L}=\frac{1}{8 \pi} \partial_{\mu} \phi \partial^{\mu} \phi=\frac{1}{8 \pi}\left[\left(\frac{\partial \phi}{\partial c t}\right)^{2}-\left(\frac{\partial \phi}{\partial x}\right)^{2}\right]
$$

From the Lagrangian (5), it follows that the wave equation $\partial_{\mu}\left[\partial \mathcal{L} / \partial\left(\partial_{\mu} \phi\right)\right]-\partial \mathcal{L} / \partial \phi=0$ for the field is

$$
0=\frac{\partial^{2} \phi}{\partial(c t)^{2}}-\frac{\partial^{2} \phi}{\partial x^{2}}
$$

while the stress-energy-momentum tensor is $\mathcal{T}^{\mu \nu}=\left[\partial \mathcal{L} / \partial\left(\partial_{\mu} \phi\right)\right] \partial^{\nu} \phi-g^{\mu \nu} \mathcal{L}$ so that the energy density is

$$
u=\mathcal{T}^{00}=\frac{1}{8 \pi}\left[\frac{1}{c^{2}}\left(\frac{\partial \phi}{\partial t}\right)^{2}+\left(\frac{\partial \phi}{\partial x}\right)^{2}\right]
$$

and the momentum density is

$$
\mathcal{T}^{01}=-\frac{1}{4 \pi} \frac{\partial \phi}{\partial c t} \frac{\partial \phi}{\partial x}
$$

In $n$ spacetime dimensions, the energy density corresponding to Eq. (17) would have the dimensions of energy $/\left(\right.$ lengt $\left.h^{n-1}\right)$. Therefore the square of the field $\phi^{2}$ has dimensions of energy $/\left(\right.$ length $\left.^{n-3}\right)$. We will consider a scalar field in two spacetime dimensions $n=2$ where energy can be measured in units of inverse length and the square of the field $\phi^{2}$ involves energy times length and so can be treated as dimensionless. 
By introducing the coordinate transformations of Eqs. (21) and (3) into the wave equation (6) together with the assumption of scalar behavior $\phi(c t, x)=\varphi(\eta, \xi)$ for the relativistic radiation field in two spacetime dimensions, we can obtain the wave equation for the radiation field $\varphi(\eta, \xi)$ in the Rindler frame as [2]

$$
0=\frac{1}{\xi^{2}} \frac{\partial^{2} \varphi}{\partial \eta^{2}}-\frac{\partial^{2} \varphi}{\partial \xi^{2}}-\frac{1}{\xi} \frac{\partial \varphi}{\partial \xi}
$$

\section{Normal Modes}

The normal modes of the scalar field $\phi_{k}$ are solutions of the wave equation with harmonic time dependence

$$
\phi_{k}(c t, x)=\operatorname{Re} \phi_{(k)}(x) \exp [-i|k| c t]
$$

In an inertial frame, the normal modes of Eq. (6) are solutions of the scalar Helmholtz equation

$$
\frac{\partial^{2} \phi_{(k)}}{\partial x^{2}}+k^{2} \phi_{(k)}=0
$$

which can be chosen as $\phi_{(k)}(x)=\exp [i k x]$, so that the general normal mode involves running wave solutions

$$
\phi(c t, x)=\operatorname{Re} \exp [i k x-i|k| c t]
$$

where $k$ can be positive or negative..

In the Rindler frame, the normal modes $\varphi_{\kappa}$ of the wave equation will not take the simple running wave form of Eq. (12). The spatial dependence is more complicated and must satisfy

$$
0=+\frac{\partial^{2} \varphi_{(\kappa)}}{\partial \xi^{2}}+\frac{1}{\xi} \frac{\partial \varphi_{(\kappa)}}{\partial \xi}+\frac{1}{\xi^{2}} \kappa^{2} \varphi_{(\kappa)}
$$

with solution $\varphi_{(\kappa)}(\xi)=\xi^{ \pm i \kappa}=\exp [ \pm i \kappa \ln \xi]$, so that in the Rindler frame the general normal mode in two spacetime dimensions is

$$
\varphi_{\kappa}(\eta, \xi)=\operatorname{Re} \exp [i \kappa \ln \xi-i|\kappa| \eta]
$$

where the parameter $\kappa$ can be positive or negative corresponding to waves moving in either the positive or negative direction. 


\section{E. Time-Dilating Conformal Transformations}

When discussing thermal radiation, we will be interested in time-dilating conformal transformations which carry radiation normal modes into normal modes. Now conformal transformations of the metric[10] involve those mappings $x^{\mu} \rightarrow \bar{x}^{\mu}=h^{\mu}\left(x^{\nu}\right)$ which transform the metric in the form

$$
g_{\mu \nu}\left(x^{\alpha}\right)=\Omega^{2}\left(\bar{x}^{\beta}\right) g_{\mu \nu}\left(\bar{x}^{\beta}\right)
$$

These transformations leave the wave equation invariant for a tensor field. However, we are not interested in conformal transformations of the metric or a change in coordinate system. Rather we will want to maintain the coordinate system and the metric while making an active transformation to a new radiation field in the original coordinate system.

A time-dilating conformal transformation takes the time coordinate into a multiple $\sigma$ of itself, and requires that the transformation of the spatial coordinates are such that the wave equation remains invariant and the metric transforms as in Eq. (15). In an inertial frame in two spacetime dimensions, a time-dilating conformal transformation is simply a uniform dilatation of both the time and space coordinates, $t \rightarrow \bar{t}=\sigma t, x \rightarrow \bar{x}=\sigma x$, while the radiation field $\phi$ transforms as a scalar field under the coordinate transformation $\bar{\phi}(c \bar{t}, \bar{x})=\phi(c t, x)$. This transformation carries the wave equation into itself

$$
\frac{1}{c^{2}} \frac{\partial^{2} \bar{\phi}}{\partial \bar{t}^{2}}-\frac{\partial^{2} \bar{\phi}}{\partial \bar{x}^{2}}=\frac{1}{\sigma^{2}}\left(\frac{1}{c^{2}} \frac{\partial^{2} \phi}{\partial t^{2}}-\frac{\partial^{2} \phi}{\partial x^{2}}\right)=0
$$

and carries plane waves of frequency $c|k|$ into plane waves of frequency $c|k| / \sigma$ as

$$
\begin{aligned}
\bar{\phi}(c \bar{t}, \bar{x}) & =\phi(c t, x) \\
& =\phi(c \bar{t} / \sigma, \bar{x} / \sigma) \\
& =\operatorname{Re} \exp \left[i \frac{k}{\sigma} \bar{x}-i\left|\frac{k}{\sigma}\right| c \bar{t}\right]
\end{aligned}
$$

This uniform dilation corresponds to a conformal transformation in an inertial frame since

$$
d s^{2}=c^{2} d t^{2}-d x^{2}=\sigma^{-2}\left[c^{2} d \bar{t}^{2}-d \bar{x}^{2}\right]
$$

However, we note that the new radiation function $\bar{\phi}$ in Eq. (17) can be regarded as a function of the old time and space coordinates $\bar{\phi}(c t, x)=\operatorname{Re} \exp [i(k / \sigma) x-i(|k| / \sigma) c t)$ giving a new radiation field in the old coordinate system. We are interested in time-dilating 
conformal changes in this sense of mapping normal modes into new normal modes without any change in the coordinate system. Thus the time-dilating conformal transformation can be regarded as mapping the normal mode at frequency $c|k|$ into the normal mode at frequency $c|\sigma k|$ according to

$$
\phi_{k}(c t, x) \rightarrow \phi_{\sigma k}(c t, x)=\phi_{k}(c \sigma t, \sigma x)=\operatorname{Re} \exp [i \sigma k x-i|\sigma k| c t]
$$

If the positive dilatation constant $\sigma$ is chosen larger than $1, \sigma>1$, then the energy density of the transformed normal mode $\phi_{\sigma k}(c t, x)$ will be larger than the energy density of the original normal mode $\phi_{k}(c t, x)$.

We emphasize that the coordinate transformation from an inertial frame to a Rindler frame is not conformal, and the wave equation (91) in a Rindler frame is not the same as the wave equation (6) in an inertial frame.

It is clear that in an inertial frame, a space-dilating conformal transformation coincides with a time-dilating conformal transformation; both correspond to uniform dilations of all

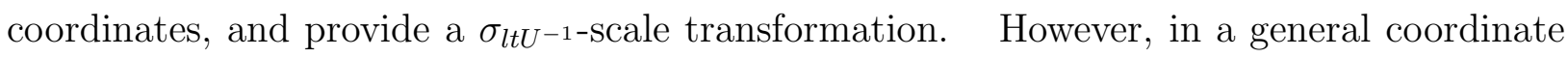
frame, a time-dilating conformal transformation is different from a space-dilating conformal transformation. Thus for a Rindler frame in two spacetime dimensions, a space-dilating conformal transformation involves the transformation

$$
\eta \rightarrow \bar{\eta}=\eta, \quad \xi \rightarrow \bar{\xi}=\sigma \xi, \quad \varphi \rightarrow \bar{\varphi}(\bar{\eta}, \bar{\xi})=\varphi(\eta, \xi),
$$

giving the metric form

$$
d s^{2}=c^{2} d t^{2}-d x^{2}=\xi^{2} d \eta^{2}-d \xi^{2}=\sigma^{-2}\left(\bar{\xi}^{2} d \bar{\eta}^{2}-d \bar{\xi}^{2}\right)
$$

Under the space-dilating conformal transformation (20), we find the wave equation transforms as

$$
\begin{aligned}
\frac{1}{\bar{\xi}^{2}} \frac{\partial^{2} \bar{\varphi}}{\partial \bar{\eta}^{2}}-\frac{\partial^{2} \bar{\varphi}}{\partial \bar{\xi}^{2}}-\frac{1}{\bar{\xi}} \frac{\partial \bar{\varphi}}{\partial \bar{\xi}} & =\frac{1}{(\sigma \xi)^{2}} \frac{\partial^{2} \varphi}{\partial \eta^{2}}-\frac{\partial^{2} \varphi}{\partial(\sigma \xi)^{2}}-\frac{1}{(\sigma \xi)} \frac{\partial \varphi}{\partial(\sigma \xi)} \\
& =\frac{1}{\sigma^{2}}\left(\frac{1}{\xi^{2}} \frac{\partial^{2} \varphi}{\partial \eta^{2}}-\frac{\partial^{2} \varphi}{\partial \xi^{2}}-\frac{1}{\xi} \frac{\partial \varphi}{\partial \xi}\right)=0
\end{aligned}
$$

giving the mapping of normal modes as

$$
\begin{aligned}
\bar{\varphi}(\bar{\eta}, \bar{\xi}) & =\varphi(\eta, \xi)=\varphi(\bar{\eta}, \bar{\xi} / \sigma) \\
& =\operatorname{Re} \exp [i \kappa \ln (\bar{\xi} / \sigma)-i|\kappa| \bar{\eta}] \\
& =\operatorname{Re} \exp [i \kappa \ln \bar{\xi}-i|\kappa| \bar{\eta}-i \kappa \ln \sigma]
\end{aligned}
$$


which is merely a change in the phase of the normal mode.

On the other hand, a time-dilating conformal transformation corresponds to

$$
\eta \rightarrow \bar{\eta}=\sigma \eta, \quad \xi \rightarrow \bar{\xi}=\xi^{\sigma}, \quad \varphi \rightarrow \bar{\varphi}(\bar{\eta}, \bar{\xi})=\varphi(\eta, \xi)
$$

giving the metric form

$$
d s^{2}=c^{2} d t^{2}-d x^{2}=\xi^{2} d \eta^{2}-d \xi^{2}=\left(\bar{\xi}^{(1 / \sigma-1)} / \sigma\right)^{2}\left(\bar{\xi}^{2} d \bar{\eta}^{2}-d \bar{\xi}^{2}\right)
$$

Under a time-dilating conformal transformation (24), we find the wave equation transforms as

$$
\begin{aligned}
\frac{1}{\bar{\xi}^{2}} \frac{\partial^{2} \bar{\varphi}}{\partial \bar{\eta}^{2}}-\frac{\partial^{2} \bar{\varphi}}{\partial \bar{\xi}^{2}}-\frac{1}{\bar{\xi}} \frac{\partial \bar{\varphi}}{\partial \bar{\xi}} & =\frac{1}{\left(\xi^{\sigma}\right)^{2}} \frac{\partial^{2} \varphi}{\partial \eta^{2}}-\frac{\partial^{2} \varphi}{\partial\left(\xi^{\sigma}\right)^{2}}-\frac{1}{\left(\xi^{\sigma}\right)} \frac{\partial \varphi}{\partial\left(\xi^{\sigma}\right)} \\
& =\frac{1}{\sigma^{2} \xi^{2 \sigma-2}}\left(\frac{1}{\xi^{2}} \frac{\partial^{2} \varphi}{\partial \eta^{2}}-\frac{\partial^{2} \varphi}{\partial \xi^{2}}-\frac{1}{\xi} \frac{\partial \varphi}{\partial \xi}\right)=0
\end{aligned}
$$

giving the mapping of normal modes $\varphi \rightarrow \bar{\varphi}$ as

$$
\begin{aligned}
\bar{\varphi}(\bar{\eta}, \bar{\xi}) & =\varphi(\eta, \xi)=\varphi\left(\bar{\eta} / \sigma, \bar{\xi}^{1 / \sigma}\right) \\
& =\operatorname{Re} \exp \left[i \kappa \ln \left(\bar{\xi}^{1 / \sigma}\right)-i|\kappa| \bar{\eta} / \sigma\right] \\
& =\operatorname{Re} \exp \left[i \frac{\kappa}{\sigma} \ln \bar{\xi}-i\left|\frac{\kappa}{\sigma}\right| \bar{\eta}\right]
\end{aligned}
$$

which corresponds to a change in the "frequency" of the normal mode in the Rindler frame. Again, we are interested in time-dilating conformal transformations only in the sense of carrying normal mode solutions into normal mode solutions. Thus the radiation field $\bar{\varphi}$ in Eq. (27) can be regarded as a new radiation field in the original coordinates $\bar{\varphi}(\eta, \xi)=$ $\operatorname{Re} \exp [i(\kappa / \sigma) \ln \xi-i|\kappa / \sigma| \eta]$ in the original coordinate frame. We will treat a time-dilating conformal transformation in a Rindler frame as the mapping of a Rindler normal mode $\varphi_{\kappa}(\eta, \xi)$ into a new normal mode $\varphi_{\sigma \kappa}(\eta, \xi)$ in the same coordinate frame according to

$$
\varphi_{\kappa}(\eta, \xi) \rightarrow \varphi_{\sigma \kappa}(\eta, \xi)=\varphi_{\kappa}\left(\sigma \eta, \xi^{\sigma}\right)=\operatorname{Re} \exp [i(\sigma k) \xi-i|\sigma \kappa| \eta]
$$

If the positive constant $\sigma$ exceeds $1, \sigma>1$, then the energy density of the transformed normal mode $\varphi_{\sigma \kappa}(\eta, \xi)$ is larger than the energy density of the initial normal mode $\varphi_{\kappa}(\eta, \xi)$. 


\section{ZERO-POINT RADIATION}

\section{A. Casimir Forces and Classical Zero-Point Radiation}

Experimental measurements of Casimir forces between conductors can be described theoretically in terms of forces due to random classical radiation. Experiment shows [12] that there are forces between uncharged conductors which can be accounted for by assuming the presence of random classical electromagnetic radiation even at zero temperature. [13] The spectrum of this zero-point radiation is uniquely determined by the assumption that the spectrum of random classical radiation is Lorentz invariant[14] in an inertial frame or,

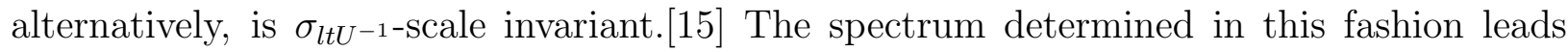
to theoretically-predicted forces in agreement with the experimentally-measured separationdependence of the Casimir forces, and the one unknown scale factor in the classical zero-point radiation spectrum is chosen to fit the magnitude of the experimental measurements. It is natural to expect that thermal radiation at non-zero temperature $T>0$ fits smoothly with the zero-point radiation at $T=0$. We will make this smooth transition under time-dilating conformal transformations the basis for obtaining the thermal radiation spectrum within classical physics.

\section{B. Zero-Point Radiation in a General Spacetime}

It is a familiar idea that relativistic radiation contain no intrinsic lengths, times, or energies, and so can be introduced into a general spacetime. Classical zero-point radiation is random classical radiation which has a scale-invariant spectrum. Since the radiation includes no intrinsic lengths and the zero-point radiation spectrum also involves no intrinsic lengths, the two-field correlation function for zero-point radiation at spacetime points $P$ and $Q$ must involve only the distance along the geodesic curve connecting $P$ and $Q$, with a scale factor to be determined by experiment. The scale factor is used to fit the experimentallyobserved Casimir forces between conductors which can be calculated within classical physics based upon the classical electromagnetic zero-point radiation spectrum. Within an inertial frame with its geodesic coordinates, the spacetime separation squared between the spacetime points $P$ and $Q$ is given by $s_{P Q}^{2}=c^{2}\left(t_{P}-t_{Q}\right)^{2}-\left(\mathbf{r}_{P}-\mathbf{r}_{Q}\right)^{2}$, and the dimensionality of the fields will determine the zero-point correlation function up to a multiplicative constant. 
Below we illustrate these ideas for a scalar field in two spacetime dimensions.

\section{Zero-Point Radiation in an Inertial Frame}

At this point, we obtain the forms taken by zero-point radiation in an inertial frame and in a Rindler frame before we consider the transition to thermal radiation. Zero-point radiation, and indeed thermal radiation at any temperature, involves random radiation whose character does not change in time. In any coordinate frame in which random radiation is a stationary distribution, the radiation field can be expressed in terms of a sum over the normal modes with random phases between the normal modes.[16] In an inertial frame in two spacetime dimensions, the correlation function for time-stationary random radiation can be evaluated using plane wave normal modes with random phases and requiring Lorentz invariance or scale invariance. Thus a periodic normal mode $\phi_{k}(c t, x)$ of the scalar radiation field in a box of length $l$ for $k=2 n \pi / l$ (positive or negative) can be written from Eqs. (12) and (7) as

$$
\phi_{k}(c t, x)=\left(\frac{8 \pi U(|k|)}{l}\right)^{1 / 2} \frac{1}{|k|} \cos [k x-|k| c t+\theta(k)]
$$

where $U(|k|)$ is the energy of the normal mode of angular frequency $\omega=c|k|$, and where $\theta(k)$ is a random phase which is distributed uniformly on the interval $[0,2 \pi)$ and is distributed independently for each vector $k$. In the infinite-length limit, the random radiation field for isotropic radiation can then be written as

$$
\phi(c t, x)=2 \int_{-\infty}^{\infty} d k \frac{U^{1 / 2}(|k|)}{|k|} \cos [k x-|k| c t+\theta(k)]
$$

When averaged over the random phases $\theta(k)$, we find[17]

$$
<\cos \theta(k) \cos \theta\left(k^{\prime}\right)>=<\sin \theta(k) \sin \theta\left(k^{\prime}\right)>=\frac{1}{2} \delta\left(k-k^{\prime}\right)
$$

and

$$
<\sin \theta(k) \cos \theta\left(k^{\prime}\right)>=0
$$

It follows that the two-field correlation function for isotropic random radiation takes the form[2]

$$
<\phi(c t, x) \phi\left(c t^{\prime}, x^{\prime}\right)>=2 \int_{-\infty}^{\infty} d k \frac{U(|k|)}{k^{2}} \cos \left[k\left(x-x^{\prime}\right)-|k| c\left(t-t^{\prime}\right)\right]
$$

If we assume that the two-field correlation function is for zero-point radiation $\phi_{0}$, and so is

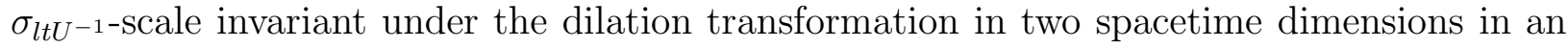


inertial frame where the square of the field $\phi^{2}$ must transform as an invariant scalar field, then we require

$$
<\phi_{0}(c \sigma t, \sigma x) \phi_{0}\left(c \sigma t^{\prime}, \sigma x^{\prime}\right)>=<\phi_{0}(c t, x), \phi_{0}\left(c t^{\prime}, x^{\prime}\right)>
$$

In terms of the spectrum $U(|k|)$ appearing in Eq. (30), the scale-invariance requirement is that

$$
\begin{aligned}
& <\phi_{0}(c \sigma t, \sigma x) \phi_{0}\left(c \sigma t^{\prime}, \sigma x^{\prime}\right)> \\
& =2 \int d k \frac{U(|k|)}{k^{2}} \cos \left[k \sigma\left(x-x^{\prime}\right)-|k| c \sigma\left(t-t^{\prime}\right)\right] \\
& =2 \int \sigma d k \frac{\sigma U(|k|)}{(\sigma k)^{2}} \cos \left[k \sigma\left(x-x^{\prime}\right)-|k| c \sigma\left(t-t^{\prime}\right)\right] \\
& =2 \int d \bar{k} \frac{(\sigma U(|\bar{k} / \sigma|)}{|\bar{k}|^{2}} \cos \left[\bar{k}\left(x-x^{\prime}\right)-|\bar{k}| c\left(t-t^{\prime}\right)\right]
\end{aligned}
$$

should agree with $<\phi_{0}(c t, x) \phi_{0}\left(c t^{\prime}, x^{\prime}\right)>$ as in Eq.(33). This requirement means that

$$
\sigma U(|k| / \sigma)=U(|k|)
$$

for all $k$, which corresponds to $U(|k|)$ being linear in $|k|$,

$$
U(|k|)=\mathfrak{c o n s t} \times|k| .
$$

where $\mathfrak{c o n s t}$ is the multiplicative constant setting the scale of classical electromagnetic zeropoint radiation. This spectrum is also Lorentz invariant[2] so that zero-point radiation takes the same spectral form in all inertial frames.

Having now found the spectrum (37) for zero-point radiation in two spacetime dimensions, we would like to evaluate the two-field correlation function (33) for zero-point radiation in closed form. However, the correlation function is divergent at small values of $k$. In order to avoid this divergence, we will follow Davies and Fulling[18] and will consider the derivatives of the correlation function which take the form[2]

$$
\begin{aligned}
& <\phi_{0}(c t, x) \partial_{c t^{\prime}} \phi_{0}\left(c t^{\prime}, x^{\prime}\right)>=2 \int d k\left(\frac{\mathfrak{c o n s t}}{|k|}\right)(-|k|) \sin \left[\left\{k\left(x-x^{\prime}\right)-|k| c\left(t-t^{\prime}\right)\right]\right. \\
& =\mathfrak{c o n s t} \times \frac{-4 c\left(t-t^{\prime}\right)}{\left(x-x^{\prime}\right)^{2}-c^{2}\left(t-t^{\prime}\right)^{2}}
\end{aligned}
$$

and

$$
\begin{aligned}
& <\phi_{0}(c t, x) \partial_{x^{\prime}} \phi_{0}\left(c t^{\prime}, x^{\prime}\right)>=2 \int d k\left(\frac{\mathfrak{c o n s t}}{|k|}\right)(k) \sin \left[\left\{k\left(x-x^{\prime}\right)-|k| c\left(t-t^{\prime}\right)\right]\right. \\
& =\mathfrak{c o n s t} \times \frac{4\left(x-x^{\prime}\right)}{\left(x-x^{\prime}\right)^{2}-c^{2}\left(t-t^{\prime}\right)^{2}}
\end{aligned}
$$


Here the integrals involve a singular Fourier sine transform which is evaluated using a temporary cut-off at high frequency. [2]

\section{Zero-Point Radiation in a Rindler Frame}

Now we wish to consider zero-point radiation in a Rindler frame. Since the relativistic scalar field is a scalar under coordinate transformations, taking the same value at the same spatial point, $\varphi(\eta, \xi)=\phi(c t, x)=\phi(\xi \sinh \eta, \xi \cosh \eta)$, we can obtain the two-field correlation function for the scalar field simply by carrying out a coordinate transformation

$$
\begin{aligned}
& <\varphi_{0}(\eta, \xi) \partial_{\eta^{\prime}} \varphi_{0}\left(\eta^{\prime}, \xi^{\prime}\right)> \\
& =<\phi_{0}(c t, x) \partial_{c t^{\prime}} \phi_{0}\left(c t^{\prime}, x^{\prime}\right)>\frac{\partial c t^{\prime}}{\partial \eta^{\prime}}+<\phi_{0}(c t, x) \partial_{x^{\prime}} \phi_{0}\left(c t^{\prime}, x^{\prime}\right)>\frac{\partial x^{\prime}}{\partial \eta^{\prime}} \\
& =\operatorname{const} \times \frac{-4 c\left(t-t^{\prime}\right)}{\left(x-x^{\prime}\right)^{2}-c^{2}\left(t-t^{\prime}\right)^{2}} \xi^{\prime} \cosh \eta^{\prime}+\mathfrak{c o n s t} \times \frac{4\left(x-x^{\prime}\right)}{\left(x-x^{\prime}\right)^{2}-c^{2}\left(t-t^{\prime}\right)^{2}} \xi^{\prime} \sinh \eta^{\prime} \\
& =\operatorname{const} \times \frac{4 \xi^{\prime}\left(\xi \sinh \eta-\xi^{\prime} \sinh \eta^{\prime}\right) \cosh \eta^{\prime}-4 \xi^{\prime}\left(\xi \cosh \eta-\xi^{\prime} \cosh \eta^{\prime}\right) \sinh \eta^{\prime}}{\left[\left(\xi \sinh \eta-\xi^{\prime} \sinh \eta^{\prime}\right)^{2}-\left(\xi \cosh \eta-\xi^{\prime} \cosh \eta^{\prime}\right)^{2}\right]} \\
& =\mathfrak{c o n s t} \times \frac{4 \xi \xi^{\prime} \sinh \left(\eta-\eta^{\prime}\right)}{\left[2 \xi \xi^{\prime} \cosh \left(\eta-\eta^{\prime}\right)-\xi^{2}-\xi^{\prime 2}\right]}
\end{aligned}
$$

and

$$
\begin{aligned}
& <\varphi_{0}(\eta, \xi) \partial_{\xi^{\prime}} \varphi_{0}\left(\eta^{\prime}, \xi^{\prime}\right)> \\
& =<\phi_{0}(c t, x) \partial_{c t^{\prime}} \phi_{0}\left(c t^{\prime}, x^{\prime}\right)>\frac{\partial c t^{\prime}}{\partial \xi^{\prime}}+<\phi_{0}(c t, x) \partial_{x^{\prime}} \phi_{0}\left(c t^{\prime}, x^{\prime}\right)>\frac{\partial x^{\prime}}{\partial \xi^{\prime}} \\
& =\operatorname{const} \times \frac{-4 c\left(t-t^{\prime}\right)}{\left(x-x^{\prime}\right)^{2}-c^{2}\left(t-t^{\prime}\right)^{2}} \sinh \eta^{\prime}+\operatorname{const} \times \frac{4\left(x-x^{\prime}\right)}{\left(x-x^{\prime}\right)^{2}-c^{2}\left(t-t^{\prime}\right)^{2}} \cosh \eta^{\prime} \\
& =\operatorname{const} \times \frac{4\left(\xi \sinh \eta-\xi^{\prime} \sinh \eta^{\prime}\right) \sinh \eta^{\prime}-4\left(\xi \cosh \eta-\xi^{\prime} \cosh \eta^{\prime}\right) \cosh \eta^{\prime}}{\left[\left(\xi \sinh \eta-\xi^{\prime} \sinh \eta^{\prime}\right)^{2}-\left(\xi \cosh \eta-\xi^{\prime} \cosh \eta^{\prime}\right)^{2}\right.} \\
& =\mathfrak{c o n s t} \times \frac{4\left[\xi^{\prime}-\xi \cosh \left(\eta-\eta^{\prime}\right)\right]}{\left[2 \xi \xi^{\prime} \cosh \left(\eta-\eta^{\prime}\right)-\xi^{2}-\xi^{\prime 2}\right]}
\end{aligned}
$$

We notice immediately that this correlation function is time stationary since it depends only upon the time difference $\eta-\eta^{\prime}$ and not on the individual times $\eta$ and $\eta^{\prime}$. Indeed we expect that zero-point radiation is the only spectrum of random radiation which is time stationary in both all inertial frames and in all Rindler frames. 


\section{THERMAL RADIATION}

\section{A. Thermal Radiation at $T>0$ is Coordinate-Frame Dependent}

Although the correlation function for classical zero-point radiation can be specified in a coordinate-free manner as involving the separation along a geodesic curve between the spacetime points where the fields are evaluated, thermal radiation at non-zero temperature $T>0$ requires a specific coordinate frame. Thus although zero-point radiation is Lorentzinvariant in an inertial frame, thermal radiation at $T>0$ involves a finite (not divergent) density of additional energy above the divergent zero-point spectrum, and so is associated with a unique coordinate frame, namely the frame in which the random radiation is timestationary and in which radiation is isotropic at every spatial point.

\section{B. Thermal Radiation and Inertial-Frame Dilatations of the Conformal Group}

In an inertial frame, the pattern of thermal radiation depends upon the one parameter of temperature $T$. Since the conformal transformations are symmetry transformations of electromagnetism, we might expect some interesting transformations when conformal transformations are applied to the thermal radiation spectrum. In an inertial frame, the dilatations of the conformal group are uniform scale transformations of length, time, and energy while the proper conformal transformations can be regarded as local spacetimedependent scale transformations. [7] Under dilatations of the conformal group in an inertial frame, thermal radiation at temperature $T>0$ is carried into thermal radiation at a new temperature $T / \sigma$ which is a positive multiple of the old temperature and which can approach ever close to zero temperature as the positive constant $\sigma \rightarrow \infty$. However, once zero temperature is reached in an inertial frame, the process can not be reversed by use of the dilatations of the conformal group; one can not map zero-point radiation into non-zero thermal radiation by conformal-group dilatations in an inertial frame.

This scale invariance of zero-point radiation in an inertial frame was discussed in Section III C, but here we wish to reiterate the invariance in connection with the closed form expressions for the correlation functions given in (38) and (39). Thus the invariance follows from $\partial t / \partial(\sigma t)=1 / \sigma$ and $\partial x / \partial(\sigma x)=1 / \sigma$, where 


$$
\begin{aligned}
& <\phi_{0}(c \sigma t, \sigma x) \partial_{c \sigma t^{\prime}} \phi_{0}\left(c \sigma t^{\prime}, \sigma x^{\prime}\right)>=\mathfrak{c o n s t} \frac{-4 c\left(\sigma t-\sigma t^{\prime}\right)}{\left(\sigma x-\sigma x^{\prime}\right)^{2}-c^{2}\left(\sigma t-\sigma t^{\prime}\right)^{2}} \\
& =\frac{1}{\sigma} \mathfrak{c o n s t} \frac{-4 c\left(t-t^{\prime}\right)}{\left(x-x^{\prime}\right)^{2}-c^{2}\left(t-t^{\prime}\right)^{2}}=\frac{1}{\sigma}<\phi_{0}(c t, x) \partial_{c t^{\prime}} \phi_{0}\left(c t^{\prime}, x^{\prime}\right)> \\
& <\phi_{0}(c \sigma t, \sigma x) \partial_{x^{\prime}} \phi_{0}\left(c \sigma t^{\prime}, \sigma x^{\prime}\right)>=\mathfrak{c o n s t} \frac{4\left(\sigma x-\sigma x^{\prime}\right)}{\left(\sigma x-\sigma x^{\prime}\right)^{2}-c^{2}\left(\sigma t-\sigma t^{\prime}\right)^{2}} \\
& =\frac{1}{\sigma} \mathfrak{c o n s t} \frac{4\left(x-x^{\prime}\right)}{\left(x-x^{\prime}\right)^{2}-c^{2}\left(t-t^{\prime}\right)^{2}}=\frac{1}{\sigma}<\phi_{0}(c t, x) \partial_{c x^{\prime}} \phi_{0}\left(c t^{\prime}, x^{\prime}\right)>
\end{aligned}
$$

We see again that zero-point radiation is completely invariant under the dilatations of the conformal group in an inertial frame.

\section{Contrasting Behavior for Thermal Radiation in Inertial and Non-Inertial}

\section{Frames}

An inertial frame is a very special system, not only as far as geodesic coordinates are concerned but also as far as thermal radiation is concerned. An inertial frame has no intrinsic lengths or times which are associated with the spatial points of the frame. Accordingly, in equilibrium, the temperature, pressure, energy density, and field correlations are everywhere the same throughout the entire spacetime. The situation is completely different in a noninertial frame, for example in a Rindler frame. In a non-inertial frame, the temperature at equilibrium will in general be a local function of the spatial coordinates, just as the local proper time at a fixed coordinate is a function of the spatial coordinates and the time parameter. Thus in a Rindler frame, the equilibrium temperature $T$ is not constant but rather varies inversely as the distance $\xi$ to the event horizon; the product $T \xi$ is a constant, and from Eq. (4) in a Rindler frame, the proper time interval $d \tau$ at a fixed spatial coordinate $\xi$ is given by $c d \tau=d s=\xi d \eta$. The contrast in behavior between inertial and non-inertial frames suggests the possibility that time-dilating conformal transformations may connect zero-point radiation continuously with thermal radiation at non-zero-temperature in a noninertial frame. Associated with the coordinates in a non-inertial frame will be characteristic times which are related to variations in the zero-point radiation correlation function. Under a time-dilating conformal transformation, the spectrum of zero-point radiation will be carried into a new stationary distribution of random radiation which is different from zero-point 
radiation. If the zero-point radiation spectrum is connected continuously with non-zero temperature radiation, then we expect a time-dilating conformal transformation to carry the zero-point spectrum into the thermal spectrum at non-zero-temperature in a non-inertial frame.

\section{Space-Dilating Conformal Transformations in a Rindler Frame}

The dilatations associated with conformal symmetry can be carried out easily in a Rindler frame in two spacetime dimensions. If we first consider the space-dilating conformal transformation of Eq. (20), then we find that zero-point radiation in a Rindler frame is invariant. Thus from $\partial \xi^{\prime} / \partial\left(\sigma \xi^{\prime}\right)=1 / \sigma$, we have

$$
\begin{aligned}
& <\varphi_{0}(\eta, \sigma \xi) \partial_{\eta^{\prime}} \varphi_{0}\left(\eta^{\prime}, \sigma \xi^{\prime}\right)>=\operatorname{const} \frac{4(\sigma \xi)\left(\sigma \xi^{\prime}\right) \sinh \left(\eta-\eta^{\prime}\right)}{\left[2(\sigma \xi)\left(\sigma \xi^{\prime}\right) \cosh \left(\eta-\eta^{\prime}\right)-(\sigma \xi)^{2}-\left(\sigma \xi^{\prime}\right)^{2}\right]} \\
& =\mathfrak{c o n s t} \frac{4 \xi \xi^{\prime} \sinh \left(\eta-\eta^{\prime}\right)}{\left[2 \xi \xi^{\prime} \cosh \left(\eta-\eta^{\prime}\right)-\xi^{2}-\xi^{\prime 2}\right]}=<\varphi_{0}(\eta, \xi) \partial_{\eta^{\prime}} \varphi_{0}\left(\eta^{\prime}, \xi^{\prime}\right)>
\end{aligned}
$$

while

$$
\begin{aligned}
& <\varphi_{0}(\eta, \sigma \xi) \partial_{\sigma \xi^{\prime}} \varphi_{0}\left(\eta^{\prime}, \sigma \xi^{\prime}\right)>=\mathfrak{c o n s t} \frac{4\left[\left(\sigma \xi^{\prime}\right)-(\sigma \xi) \cosh \left(\eta-\eta^{\prime}\right)\right]}{\left[2(\sigma \xi)\left(\sigma \xi^{\prime}\right) \cosh \left(\eta-\eta^{\prime}\right)-(\sigma \xi)^{2}-\left(\sigma \xi^{\prime}\right)^{2}\right]} \\
& =\mathfrak{c o n s t} \frac{4\left[\xi^{\prime}-\xi \cosh \left(\eta-\eta^{\prime}\right)\right]}{\left[2 \xi \xi^{\prime} \cosh \left(\eta-\eta^{\prime}\right)-\xi^{2}-\xi^{\prime 2}\right]}=\frac{1}{\sigma}<\varphi_{0}(\eta, \xi) \partial_{\xi^{\prime}} \varphi_{0}\left(\eta^{\prime}, \xi^{\prime}\right)>
\end{aligned}
$$

From the metric in Eq. (4), we see that the Rindler spatial coordinate $\xi$ is a geodesic coordinate and so the space-dilating conformal transformation leaves the zero-point correlation function unchanged. This invariance is consistent with the result in Eq. (23) which showed that the space-dilating conformal transformation in a Rindler frame merely changed the

phase of a radiation normal mode. Since zero-point radiation has random phases between the modes, this change in phase for each mode does not alter the correlation function for zero-point radiation in the Rindler frame.

\section{E. Time-Dilating Conformal Transformations in a Rindler Frame}

Next we consider a time-dilating conformal transformation of the zero-point radiation in the Rindler frame. The time-dilating conformal transformation is given in Eq. (24). In this case, we find

$$
<\varphi_{0}\left(\sigma \eta, \xi^{\sigma}\right) \partial_{\sigma \eta^{\prime}} \varphi_{0}\left(\sigma \eta^{\prime}, \xi^{\prime \sigma}\right)>=\operatorname{const} \frac{4 \xi^{\sigma} \xi^{\prime \sigma} \sinh \left(\sigma \eta-\sigma \eta^{\prime}\right)}{\left[2 \xi^{\sigma} \xi^{\prime \sigma} \cosh \left(\sigma \eta-\sigma \eta^{\prime}\right)-\xi^{\sigma 2}-\xi^{\prime \sigma 2}\right]}
$$




$$
<\varphi_{0}\left(\sigma \eta, \xi^{\sigma}\right) \partial_{\xi^{\prime}} \varphi_{0}\left(\sigma \eta^{\prime}, \xi^{\prime \sigma}\right)>=\mathfrak{c o n s t} \frac{4\left[\xi^{\prime \sigma}-\xi^{\sigma} \cosh \left(\sigma \eta-\sigma \eta^{\prime}\right)\right]}{\left[2 \xi^{\sigma} \xi^{\prime \sigma} \cosh \left(\sigma \eta-\sigma \eta^{\prime}\right)-\xi^{\sigma 2}-\xi^{\prime \sigma 2}\right]}
$$

These correlation functions (46) and (47) found from the time-dilating conformal transformation are not invariant but rather involve entirely new functional forms, different from the correlations for zero-point radiation in Eqs. (40) and (41).

In order to simplify the situation, we consider the correlation functions in Eqs. (46) and (47) at different times $\eta$ and $\eta^{\prime}$ but at a single spatial point $\xi=\xi^{\prime}$. The correlation function (47) involving the spatial derivative becomes

$$
<\varphi_{0}\left(\sigma \eta, \xi^{\sigma}\right) \partial_{\xi^{\prime}} \varphi_{0}\left(\sigma \eta^{\prime}, \xi^{\prime \sigma}\right)>_{\xi=\xi^{\prime}}=-\mathfrak{c o n s t} \frac{2}{\xi^{\sigma}}
$$

and is independent of the time parameter $\eta$ or $\eta^{\prime}$. The correlation function (46) involving the time derivative becomes

$$
\begin{aligned}
& <\varphi_{0}\left(\sigma \eta, \xi^{\sigma}\right) \partial_{\sigma \eta^{\prime}} \varphi_{0}\left(\sigma \eta^{\prime}, \xi^{\sigma}\right)>=\mathfrak{c o n s t} \frac{4 \xi^{\sigma} \xi^{\sigma} \sinh \left(\sigma \eta-\sigma \eta^{\prime}\right)}{\left[2 \xi^{\sigma} \xi^{\sigma} \cosh \left(\sigma \eta-\sigma \eta^{\prime}\right)-\xi^{\sigma 2}-\xi^{\sigma 2}\right]} \\
& =\mathfrak{c o n s t} \frac{4 \sinh \left[\sigma\left(\eta-\eta^{\prime}\right)\right]}{2 \cosh \left[\sigma\left(\eta-\eta^{\prime}\right)\right]-2}=\mathfrak{c o n s t} \frac{8 \sinh \left[\sigma\left(\eta-\eta^{\prime}\right) / 2\right] \cosh \left[\sigma\left(\eta-\eta^{\prime}\right) / 2\right]}{2\left\{2 \sinh ^{2}\left[\sigma\left(\eta-\eta^{\prime}\right) / 2\right]\right\}} \\
& =\mathfrak{c o n s t} \times 2 \operatorname{coth}\left[\sigma\left(\eta-\eta^{\prime}\right) / 2\right]
\end{aligned}
$$

which is independent of the spatial point $\xi$. Now as discussed in Section II E, we are interpreting the time-dilating conformal transformation as a transformation of the radiation field in the same coordinate frame. Thus in Eq. (49), this correlation function obtained from zero-point radiation by a time-dilating conformal transformation should involve the correlation function $<\varphi_{\alpha}(\eta, \xi) \varphi_{\alpha}\left(\eta^{\prime}, \xi\right)>$ at times $\eta$ and $\eta^{\prime}$ for thermal radiation at some finite non-zero temperature with temperature parameter $\alpha$, corresponding to

$$
<\varphi_{\alpha}(\eta, \xi) \varphi_{\alpha}\left(\eta^{\prime}, \xi\right)>=<\varphi_{0}\left(\sigma \eta, \xi^{\sigma}\right) \varphi_{0}\left(\sigma \eta^{\prime}, \xi^{\sigma}\right)>
$$

in analogy with the time-dilating conformal transformation in Eq. (28). If we introduce the derivative with respect to $\eta^{\prime}$ so as to make direct contact with Eq. (49), then we have

$$
\begin{aligned}
\frac{\partial}{\partial\left(\xi \eta^{\prime}\right)} & <\varphi_{\alpha}(\eta, \xi) \varphi_{\alpha}\left(\eta^{\prime}, \xi\right)>=\frac{\sigma}{\xi} \frac{\partial}{\partial\left(\sigma \eta^{\prime}\right)}<\varphi_{0}\left(\sigma \eta, \xi^{\sigma}\right) \varphi_{0}\left(\sigma \eta^{\prime}, \xi^{\sigma}\right)> \\
& =\frac{\sigma}{\xi}<\varphi_{0}\left(\sigma \eta, \xi^{\sigma}\right) \partial_{\sigma \eta^{\prime}} \varphi_{0}\left(\sigma \eta^{\prime}, \xi^{\sigma}\right)> \\
& =\operatorname{const} \frac{2 \sigma}{\xi} \operatorname{coth}\left[\sigma\left(\eta-\eta^{\prime}\right) / 2\right]=\operatorname{const} \frac{2 \sigma}{\xi} \operatorname{coth}\left(\frac{\sigma}{\xi} \frac{\left(\xi \eta-\xi \eta^{\prime}\right)}{2}\right)
\end{aligned}
$$


If we now introduce the proper time $\tau=\xi \eta / c$ at the fixed spatial point $\xi$ in the Rindler frame, then the correlation function (51) becomes

$$
\begin{aligned}
\frac{\partial}{\partial\left(\xi \eta^{\prime}\right)} & <\varphi_{\alpha}(\eta, \xi) \varphi_{\alpha}\left(\eta^{\prime}, \xi\right)>=\frac{\partial}{\partial c \tau^{\prime}}<\varphi_{\alpha}(\eta, \xi) \varphi_{\alpha}\left(\eta^{\prime}, \xi\right)> \\
& =\operatorname{const} \frac{2 \sigma}{\xi} \operatorname{coth}\left(\frac{\sigma}{\xi} \frac{\left(c \tau-c \tau^{\prime}\right)}{2}\right)
\end{aligned}
$$

where $\tau-\tau^{\prime}$ is the proper time difference between the spacetime points and $\sigma / \xi$ corresponds to some parameter giving the temperature of the radiation at the location $\xi$.

\section{F. Thermal Radiation in an Inertial Frame Obtained as a Limit from a Rindler}

\section{Frame}

We obtain the correlation function for thermal radiation in an inertial frame from the thermal radiation correlation function (52) by moving the spatial point $\xi$ ever further from the event horizon, $\xi \rightarrow \infty$, but keeping the temperature parameter $\sigma / \xi$ constant by continuously increasing $\sigma$. In the limit, we obtain the time correlation function for thermal radiation in vanishing proper acceleration $c^{2} / \xi \rightarrow 0$, which corresponds to thermal radiation in an inertial frame[2]

$$
\begin{aligned}
\frac{\partial}{\partial c t^{\prime}} & <\phi_{\alpha}(c t, x) \phi_{\alpha}\left(c t^{\prime}, x\right)> \\
& =\operatorname{con} \mathfrak{s t} \frac{2 \sigma}{\xi} \operatorname{coth}\left(\frac{\sigma}{\xi} \frac{\left(c t-c t^{\prime}\right)}{2}\right)
\end{aligned}
$$

The actual radiation spectrum is obtained from the correlation function Eq. (53) by taking the inverse Fourier time transform of the correlation function expression (33),

$$
\begin{aligned}
& <\phi_{\alpha}(c t, x) \partial_{c t^{\prime}} \phi_{\alpha}\left(c t^{\prime}, x\right)>=2 \int_{-\infty}^{\infty} d k \frac{U_{T}(|k|)}{k^{2}}(-|k|) \sin \left[k\left(x-x^{\prime}\right)-|k| c\left(t-t^{\prime}\right)\right]_{x=x^{\prime}} \\
& =2 \int_{-\infty}^{\infty} d k \frac{U_{T}(|k|)}{k^{2}}(|k|) \sin \left[|k| c\left(t-t^{\prime}\right)\right]=\operatorname{const} \frac{2 \sigma}{\xi} \operatorname{coth}\left(\frac{\sigma}{\xi} \frac{\left(c t-c t^{\prime}\right)}{2}\right)
\end{aligned}
$$

giving the energy $U_{T}(|k|)$ per normal mode of frequency $\omega=c|k|$ at some temperature $T$ as[2]

$$
U_{T}(|k|)=\operatorname{const} \times|k| \operatorname{coth}\left(\frac{|k| \pi}{(\sigma / \xi)}\right)
$$

The temperature $T$ can be obtained by going to the high-temperature or low-frequency limit where the Rayleigh-Jeans spectrum becomes valid, giving

$$
U_{T}(|k|) \rightarrow \mathfrak{c o n s t} \times|k|\left(\frac{|k| \pi}{(\sigma / \xi)}\right)^{-1}=\mathfrak{c o n s t} \frac{\sigma}{\pi \xi}=k_{B} T
$$


Thus the spectrum of thermal radiation is given by

$$
U_{T}(|k|)=\mathfrak{c o n s t} \times|k| \operatorname{coth}\left(\frac{\mathfrak{c o n s t} \times|k|}{k_{B} T}\right)
$$

Traditionally, the spectrum of blackbody thermal radiation is given in terms of Planck's constant $\hbar$ rather than in terms of the scale factor const for classical electromagnetic zeropoint radiation given in Eq. (37). Since the connection involves

$$
\mathfrak{c o n s t}=\frac{1}{2} \hbar c
$$

our equation (57) corresponds to the familiar Planck spectrum with zero-point radiation,

$$
U(\omega, T)=\frac{1}{2} \hbar \omega \operatorname{coth}\left(\frac{\hbar \omega}{2 k_{B} T}\right)=\frac{1}{2} \hbar \omega+\frac{\hbar \omega}{\exp \left[\hbar \omega /\left(k_{B} T\right)\right]-1}
$$

We emphasize that in the limit $T \rightarrow 0$, the spectrum $U(\omega, T)$ of classical random radiation in Eq. (59) does not vanish, but rather goes over to the Lorentz-invariant spectrum of classical zero-point radiation. The Planck spectrum is connected continuously with zero-point radiation. Within classical physics, this continuous evolution of the spectrum of random radiation at non-zero temperature into the zero-point radiation spectrum is consistent with the results for Casimir forces where the forces at non-zero temperature are connected smoothly with the experimentally-observed forces at low temperature.

\section{DISCUSSION}

Thermal radiation is the random radiation which appears inside an isolated enclosure, and this radiation, like all random radiation, causes Casimir forces between conducting plates and dielectric surfaces, associated with the discrete nature of radiation normal modes in restricted volumes. At high temperatures, the random thermal radiation produces Casimir forces which follow from the Rayleigh-Jeans spectrum and depend only on the temperature $T$ and the geometrical dimensions. [19] However at low temperatures as $T \rightarrow 0$, the Casimir

forces do not vanish, indicating the presence of random radiation even at zero temperature. This radiation corresponds to classical zero-point radiation. We expect a smooth evolution of the thermal radiation spectrum with temperature as the temperature is lowered, fitting with a smooth evolution of the Casimir forces at high temperature over to the Casimir forces at zero temperature. Under a time-dilating conformal transformation, thermal radiation 
is carried into itself, so that at each spatial point $T \rightarrow T^{\prime}=T / \sigma$ where $\sigma$ is a positive constant. Thermal radiation is also a situation of greatest randomness and least information. At zero temperature, the least information means that the random radiation gives no more information than is contained in the structure of spacetime itself. Thus at zero temperature, we expect that the two-field zero-point correlation function at points $P$ and $Q$ involves only the distance along a geodesic between the spacetime points. In a noninertial static coordinate frame, the time parameter is separated from the spatial coordinates so that a time-dilating conformal transformation can carry zero-point radiation into thermal radiation at non-zero temperature. This general situation is quite different from that in an inertial frame where a time-dilating conformal transformation is the same as a uniform coordinate dilation, and the two-field zero-point correlation function is invariant under a conformal transformation. Thus in an inertial frame, information about zero-point radiation does not give us any information about the thermal radiation spectrum at finite non-zero temperature. In this article, we have considered classical random scalar radiation in a Rindler frame in two spacetime dimensions. We find that a time-dilating conformal transformation carries the zero-point radiation spectrum into the thermal radiation spectrum at a non-zero temperature in a Rindler frame. The thermal radiation spectrum in the Rindler frame can be carried back to an inertial frame, giving the Planck spectrum with zero-point radiation. Thus Planck's spectrum of blackbody radiation follows from zero-point radiation and the structure of relativistic spacetime in classical physics.

\section{ACKNOWLEDGEMENT}

I would like to thank Professor Parameswaran Nair for helpful conversations regarding conformal symmetry.

[1] T. H. Boyer, "Derivation of the Planck spectrum for relativistic classical scalar radiation from thermal equilibrium in an accelerating frame," Phys. Rev. D 81, 105024 (2010).

[2] T. H. Boyer, "Classical physics of thermal scalar radiation in two spacetime dimensions," Am. J. Phys. 79, 644-656 (2011). 
[3] See for example, R. Eisberg and R. Resnick, Quantum Physics of Atoms, Molecules, Solids, Nuclei, and Particles 2nd ed. (Wiley, New York 1985).

[4] T. H. Boyer, "Blackbody radiation and the scaling symmetry of relativistic classical electron theory with classical electromagnetic zero-point radiation," Found. Phys. 40, 1102-1116 (2010).

[5] J. H. van Vleck, "The absorption of radiation by multiply periodic orbits, and its relation to the correspondence principle and the Rayleigh-Jeans law: Part II. Calculation of absorption by multiply periodic orbits," Phys. Rev. 24, 347-365 (1924). See also, T. H. Boyer, "Equilibrium of random classical electromagnetic radiation in the presence of a nonrelativistic nonlinear electric dipole oscillator," Phys. Rev. D 13, 2832-2845 (1976); "Statistical equilibrium of nonrelativistic multiply periodic classical systems and random classical electromagnetic radiation," Phys. Rev. A 18, 1228-1237 (1978).

[6] E. Cunningham, "The principle of relativity in electrodynamics and an extension thereof ," Proc. London Math. Soc. 8, 77-98 (1910); H. Bateman, "the transformation of the electrodynamical equations," Proc. London Math. Soc. 8, 223-264 (1910).

[7] H. A. Kastrup, "Zur physikalischen Deuting und darstellungstheoretischen Analyse der konformen Transformationen der Raum und Zeit," Ann. Phys. (Leipzig) 9, 388-428 (1962).

[8] See the discussion by T. H. Boyer, "Scaling symmetry and thermodynamic equilibrium for classical electromagnetic radiation," Found. Phys. 19, 1371-1383 (1989), and also in ref. 2.

[9] See for example, W. Rindler, Essential Relativity: Special, General, and Cosmological 2nd ed. (Springer-Verlag, New York 1977), pp. 49-51. See also, See for example, B. F. Schutz, A First Course in General Relativity (Cambridge U. Press 1986), p. 150.

[10] T. Fulton, F. Rohrlich, and L. Witten, "Conformal invariance in physics," Rev. Mod. Phys. 34, 442-457 (1962).

[11] It has been pointed out by Kastrup in ref. 7 that a conformal transformation in an inertial

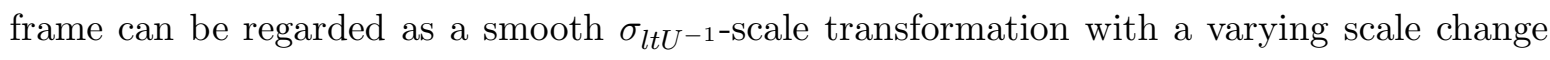
from point to point. This idea is consistent with the observation that relativistic radiation determines the metric of a spacetime only up to a scale which can vary continuously from point to point.

[12] M. J. Sparnaay, "Measurement of the attractive forces between flat plates," Physica (Amsterdam) 24, 751-764 (1958); S. K. Lamoreaux, "Demonstration of the Casimir force in the 0.6 to 
$6 \mu \mathrm{m}$ range," Phys. Rev. Lett. 78, 5-8 (1997): 81, 5475-5476 (1998); U. Mohideen, "Precision measurement of the Casimir force from 0.1 to $0.9 \mu \mathrm{m}$," ibid. 81, 4549-4552 (1998); H. B. Chan, V. A. Aksyuk, R. N. Kleinman, D. J. Bishop, and F. Capasso, "Quantum mechanical actuation of microelectromechanical systems by the Casimir force," Science 291, 1941-1944 (2001): G. Bressi, G. Caarugno, R. Onofrio, and G. Ruoso, "Measurement of the Casimir force between parallel metallic surfaces," Phys. Rev. Lett. 88, 041804(4) (2002).

[13] The original calculation was made in terms of the zero-point energy of quantum field theory by H. B. G. Casimir, "On the attraction between two perfectly conducting plates," Proc. Ned. Akad. Wetenschap. 51, 793-795 (1948). However, the same results appear in classical electrodynamics which includes classical electromagnetic zero-point radiation. See for example, T. H. Boyer, "Random electrodynamics: The theory of classical electrodynamics with classical electromagnetic zero-point radiation," Phys. Rev. 11, 790-808 (1976).

[14] T. W. Marshall, "Statistical Electrodynamics," Proc. Camb. Phil. Soc. 61, 537-546 (1965); T. H. Boyer, "Derivation of the Blackbody Radiation Spectrum without Quantum Assumptions," Phys. Rev. 182, 1374-11383 (1969).

[15] T. H. Boyer, "Conformal Symmetry of Classical Electromagnetic Zero-Point Radiation," Found. Phys. 19, 349-365 (1989).

[16] S. O. Rice, in Selected Papers on Noise and Stochastic Processes, edited by N. Wax (Dover, New York 1954), p. 133.

[17] Here we are assuming the infinite-length limit.

[18] S. A. Fulling and P. C. W. Davies, "Radiation from a moving mirror in two dimensional space-time: conformal anomaly," Proc. R. Soc. Lond. A. 348, 393-414 (1976), p. 407.

[19] T. H. Boyer, "Temperature dependence of Van der Waals forces in classical electrodynamics with classical electromagnetic zero-point radiation," Phys. Rev. A 11, 1650-1663 (1975). 